\title{
Avaliação de impacto do Programa de Fortalecimento e Melhoria da Qualidade dos Hospitais do SUS/ Minas Gerais (Pro-Hosp)
}

\author{
Kenya Valeria Micaela de Souza Noronha \\ Universidade Federal de Minas Gerais (UFMG)
}

Fátima Beatriz Carneiro Teixeira Pereira Fortes

Fundação João Pinheiro (FJP)

Mirela Castro Santos Camargos

Fundação João Pinheiro (FJP)

Danielle Ramos de Miranda Pereira

Fundação João Pinheiro (FJP)

Avaliação de impacto do Programa de Fortalecimento e Melhoria da Qualidade dos Hospitais do SUS/Minas Gerais (Pro-Hosp)

Este estudo avaliou o impacto do Programa de Fortalecimento e Melhoria da Qualidade dos Hospitais do SUS-MG (Pro-Hosp). Para tanto, foi empregado o método diferenças-emdiferenças, que permite avaliar o impacto de políticas públicas quando o indicador avaliado é observado em mais de um período no tempo. Foram selecionados quatro indicadores de desempenho hospitalar: a proporção média de cesárea, a taxa média de ocupação, a taxa média de mortalidade e o tempo médio de permanência. Constatou-se que o Pro-Hosp teve impacto na proporção média de cesáreas no sentido esperado, ou seja, de reduzi-las.

Palavras-chave: política de saúde, Sistema Único de Saúde, indicador de desempenho, avaliação de desempenho, administração estadual

Artigo recebido em novembro de 2012. Versão final em janeiro de 2014. 
Evaluación de Impacto del Programa de Fortalecimiento y Mejora de la Calidad de los Hospitales SUS /MG (Pro-Hosp)

Este estudio evaluó el impacto del Programa de Fortalecimiento y Mejora de la Calidad de los Hospitales del SUS-MG (Pro-Hosp). Por lo tanto, se empleó el método de diferencia en diferencia, lo que nos permite evaluar el impacto de las políticas públicas cuando el indicador evaluado se observa em más de um período de tiempo. Se seleccionaron cuatro indicadores de desempeño de los hospitales: la proporción media de cesáreas, la tasa media de ocupación, la tasa media de mortalidad y estancia media. Se encontró que el ProHosp impactó la proporción media de cesáreas en la dirección esperada, es decir, para reducirlas.

Palabras clave: política de salud, Sistema Único de Salud, indicador de desempeño, evaluación de desempeño, administración estadual

\section{Impact Assessment of the Program for Strenghthening and Improving the Quality of Hospitals SUS - State of Minas Gerais/Brazil (Pro-Hosp)}

This study evaluated the impact of the Program for Strengthening and Improving the Quality of Hospitals SUS-MG (Pro-Hosp). Therefore, the method employed was differencein-difference, which allows us to evaluate the impact of public policy when the indicator assessed is observed in more than one period in time. We selected four indicators of hospital performance: the average proportion of cesarean section, the average occupancy rate, the average mortality rate and average lengh of stay. It was found that the Pro-Hosp impacted the average proportion of cesarean sections in the expected direction, i.e. to reduce them.

Keywords: health policy, the Unified Health System, development indicator, developmental evaluation, local government

\section{Introdução}

Nos últimos anos, o planejamento estratégico vem ganhando cada vez mais espaço na administração pública, com a pactuação de metas, o monitoramento de indicadores e a avaliação dos resultados das políticas públicas. Desta forma, a sociedade e os próprios gestores públicos passam a ter uma maior capacidade de controle sobre as ações públicas, já que os sistemas de monitoramento e avaliação do planejamento estratégico contribuem para aperfeiçoar os mecanismos de controle da gestão (Souza, Pereira e Delgado, 2011).

Foi diante desse contexto que o Governo do Estado de Minas Gerais implementou, em 2003, o Choque de Gestão, estratégia baseada em resultados, que, entre outros aspectos, favoreceu a formulação de programas inovadores na área social (MinAs GerAIs, 2003). Visando ao aprimoramento desse modelo de gestão, em 2007 foi lançada a segunda geração do Choque de Gestão, o Estado para Resultados, que buscou aperfeiçoar a aplicação de recursos, por meio da priorização de metas e da consolidação de uma carteira de projetos estruturadores orientada para resultados (MINAs GeraIs, 2007B). 
Em sintonia com as estratégias e as orientações traçadas no Plano Mineiro de Desenvolvimento Integrado (PMDI) 2007-2023, prioridades e metas foram revistas no PMDI 2011-2030, considerando o compartilhamento de responsabilidades e a corresponsabilidade legalmente regulada, elementos centrais da revisão da estratégia de desenvolvimento de Minas Gerais (Minas Gerais, 2011). Assim, o governo buscou adotar o conceito de Estado Aberto e em Rede, operando em parceria e incorporando como pilar a gestão para a cidadania, cujo principal desafio é a participação da sociedade civil organizada na priorização e acompanhamento da implementação da estratégia governamental, sem negligenciar o equilíbrio fiscal e a busca por maior qualidade do gasto público, ambos visando à produção de melhores resultados para a população (MinAs GeRAIs, 2011).

A estratégia de desenvolvimento para os próximos 20 anos, explicitada no PMDI 2011-2030, estabelece 11 redes de desenvolvimento integrado, a saber: rede de educação e desenvolvimento humano; rede de atendimento em saúde; rede de defesa e segurança; rede de desenvolvimento social e proteção; rede de desenvolvimento econômico sustentável; rede de ciência, tecnologia e inovação; rede de desenvolvimento rural; rede de identidade mineira; rede de cidades; rede de infraestrutura; e rede de governo integrado, eficiente e eficaz. Essas redes focalizam metas-síntese, que são desdobradas em objetivos, estratégias e indicadores com metas de desempenho para medir as transformações desejadas em cada uma delas.

A rede de atenção em saúde, cuja meta-síntese é população com maior qualidade e expectativa de vida, tem como objetivos estratégicos: universalizar o acesso à atenção primária; reduzir as disparidades regionais no atendimento à saúde; consolidar as redes de atenção à saúde em todo o estado; melhorar os indicadores de morbimortalidade entre a população juvenil; e estimular maior cuidado do cidadão com a própria saúde. Entre o rol de estratégias proposto para o alcance desses objetivos, vale destacar a implementação do programa Saúde Integrada, que visa a aprimorar a gestão da rede por meio de instrumentos, ferramentas e políticas inovadoras, tais como a da Gestão do Sistema de Regulação em Saúde, a da Gestão da Política Hospitalar e a da Aquisição, Produção, Armazenamento e Distribuição de Medicamentos Básicos, Excepcionais e de Alto Custo. Mais especificamente, a Gestão da Política Hospitalar preocupa-se em prover serviços de saúde de qualidade e assistência hospitalar de importância estratégica por meio de estruturas hospitalares organizadas, e formular, acompanhar e avaliar a política de gestão hospitalar em Minas.

O objetivo deste artigo é avaliar o impacto do Programa de Fortalecimento e Melhoria da Qualidade dos Hospitais do Sistema Único de Saúde - SUS/MG 
(Pro-Hosp), instituído pela Secretaria de Saúde de Estado de Minas Gerais (SES/ MG) em maio de 2003. A formulação do Pro-Hosp vai ao encontro do que está sendo proposto pela estratégia de desenvolvimento do Estado para os próximos 20 anos, especialmente ao que se refere à Gestão da Política Hospitalar.

Para esta avaliação, foram selecionados quatro indicadores de desempenho hospitalar (proporção média de cesárea, taxa média de ocupação, taxa média de mortalidade e tempo médio de permanência), que compõem um leque extenso de metas e compromissos que os hospitais participantes do Pro-Hosp se comprometem a cumprir. A não inclusão de um número maior de indicadores, que permitiria ter uma visão mais abrangente dos resultados do Pro-Hosp, decorreu do método de análise adotado para a avaliação de impacto, que requer informações disponíveis tanto para os hospitais participantes do Pro-Hosp (grupo de tratamento), bem como para hospitais não participantes do programa localizados em Minas Gerais ou em outros estados da Federação (grupo de controle). O número limitado de indicadores avaliados decorre da inexistência de informações para os hospitais pertencentes ao grupo de controle no período de análise (de 2002 a 2008), especialmente para os localizados em outras unidades da Federação. Tem-se, portanto, que os resultados apresentados neste artigo dizem respeito à avaliação de impacto do Pro-Hosp nesses indicadores e não do programa como um todo.

A despeito dos limites dessa avaliação, ela contribui para diminuir a lacuna de estudos sobre a qualidade da oferta dos serviços hospitalares no País. Desde a criação do SUS pela Constituição Federal de 1988, inúmeros estudos têm discutido a descentralização dos serviços e os mecanismos de financiamento acoplados a ela, refletindo os avanços da política de saúde nesses aspectos. No entanto, como argumentaram La Forgia e Couttolenc (2009), as questões relacionadas à qualidade e à eficiência, especialmente de hospitais, ainda não foram adequadamente enfrentadas.

Este artigo está estruturado em mais quatro seções, além desta introdução. $\mathrm{Na}$ próxima seção é feita uma breve descrição do Pro-Hosp e seus objetivos. Em seguida, são descritas a metodologia da avaliação de impacto, a definição dos indicadores e as fontes dos dados ${ }^{1}$. A análise descritiva dos indicadores para os hospitais participantes do Pro-Hosp e os resultados da avaliação de impacto compõem a quarta seção, e o artigo finaliza com as considerações finais.

\footnotetext{
${ }^{1}$ As autoras agradecem a Naercio Aquino Menezes Filho pela contribuição para a estimação dos modelos.
} 


\section{O Programa de Fortalecimento e Melhoria da Qualidade dos Hospitais do SUS/MG (Pro-Hosp)}

O Programa de Fortalecimento e Melhoria da Qualidade dos Hospitais do SUS/ MG (Pro-Hosp) foi instituído pela Secretaria de Estado de Saúde de Minas Gerais (SES/MG), em maio de 2003. O objetivo do programa é promover a melhoria da qualidade dos hospitais integrantes do Sistema Único de Saúde (SUS) e, assim, contribuir para a consolidação da oferta de serviços hospitalares nos pólos macro e microrregionais do estado (Minas Gerais, 2007A).

Os hospitais se comprometiam a cumprir um conjunto de metas e compromissos gerenciais, assistenciais e de infra-estrutura, especificados no Plano de Ajustes e Metas (PAM), anexo ao Termo de Compromisso. Em contrapartida, a SES-MG repassaria um montante de recursos financeiros. A introdução da lógica contratual - repasse de recursos financeiros aos hospitais atrelado ao cumprimento de metas - foi o elemento inovador do programa (MinAs Gerals, 2007A).

Os recursos são provenientes do Tesouro do estado e suas aplicações são direcionadas em percentuais, sendo $40 \%$ para melhoria da qualidade da assistência, com os investimentos na manutenção e ampliação da capacidade instalada (instalações físicas e equipamentos), e 50\% livre de vinculação, podendo ser usados no aumento de qualquer uma das funções programáticas anteriores ou em custeio hospitalar. Os outros $10 \%$ devem ser aplicados na melhoria da gestão dos hospitais. De 2003 até o final de 2009, o programa beneficiou 140 hospitais (38 macrorregionais e 102 microrregionais) nas 13 macrorregiões e 75 microrregiões do estado. O valor total dos recursos repassados foi de aproximadamente 446,826 milhões de reais.

No curto prazo, os resultados esperados com o Pro-Hosp são: (a) garantia da qualidade da assistência com a readequação das instalações e (b) aumento da resolutividade da instituição com a incorporação de tecnologia e ampliação das atividades assistenciais. No médio e longo prazo, espera-se efetivar a regionalização da assistência à saúde e, consequentemente, aumentar a qualidade do atendimento à população com a resolutividade de, pelo menos, um hospital público ou filantrópico de cada microrregião em procedimentos de baixa e média complexidade (Minas Gerais, 2007A).

Entre os diversos compromissos assumidos pelos hospitais participantes do programa, existem alguns relacionados ao desempenho hospitalar. Entre eles, destaca-se: a diminuição no tempo de permanência; o aumento na taxa de ocupação; a redução na taxa de mortalidade hospitalar; a redução da taxa de cesáreas e o decréscimo da taxa de infecção hospitalar. Os quatro primeiros foram selecionados para a avaliação de impacto do Pro-Hosp. 
Os indicadores de desempenho hospitalar constituem medidas indiretas da qualidade da assistência prestada, cujo acompanhamento, de modo sistemático, permite identificar e analisar problemas na assistência hospitalar. Eles podem medir tanto os processos do cuidado ao paciente quanto seus resultados. Os processos, que são as atividades realizadas para um paciente, abarcam diversas dimensões de qualidade, como a adequação técnica do cuidado, a eficiência e a segurança, entre outros (Bittar, 2001; Travassos, Noronha e Martins, 1999). Os resultados dizem respeito às mudanças, favoráveis ou desfavoráveis, ocorridas no estado de saúde do paciente, decorrentes do processo de cuidado (Donabedian, 1980).

Embora o uso de indicadores de desempenho na avaliação da qualidade da assistência à saúde seja crescente, existem desafios que precisam ser superados para que tais indicadores reflitam adequadamente a que se propõem e possam subsidiar a tomada de decisão. Em primeiro lugar, salienta-se a escassez de dados, critérios e padrões válidos e confiáveis sobre a qualidade do cuidado, principalmente no nível do hospital. Em segundo lugar, para que uma medida de resultado possa ser considerada uma forma indireta de avaliar a qualidade do cuidado, deve-se demonstrar que ela está associada a esse processo. Por fim, de acordo com Travassos, Noronha e Martins (1999), é importante que a utilização de indicadores de desempenho seja acompanhada de estratégias de ajuste do risco, já que há fatores relacionados ao paciente que afetam o resultado, independentemente da qualidade do cuidado prestado.

Tendo em vista que os indicadores de desempenho parecem uma boa medida para avaliar a assistência e que para avaliar o impacto seria necessária uma série de informações ao longo do tempo, optou-se por utilizar quatro desses indicadores para avaliar o Pro-Hosp. Nesse caso, o impacto na taxa de infecção hospitalar não será avaliado por ausência de dados. Cabe ressaltar que os indicadores selecionados constituem apenas alguns dos inúmeros compromissos e metas que os hospitais se comprometeram a cumprir e que, portanto, os resultados devem ser compreendidos nesse contexto. Mais detalhes sobre os indicadores selecionados serão fornecidos a seguir, na metodologia.

\section{Metodologia}

Para avaliar o impacto do programa, foi empregado o método diferenças-emdiferenças, que permite avaliar o impacto de políticas públicas quando o indicador avaliado é observado em mais de um período no tempo. Estudos recentes utilizaram o método diferenças-em-diferenças para avaliar o impacto de políticas sociais (Neri; Soares, 2007; Vaitsman; Paes-Sousa, 2007; Peixoto et al., 2010; Pereira, 2011). Essa técnica consiste em comparar a variação no indicador antes e depois 
da intervenção para o grupo de tratamento (que sofreu a intervenção), com a variação observada para o grupo de controle (sem a intervenção). O método avalia as diferenças entre as médias de dois grupos e as suas respectivas diferenças ao longo do tempo. O impacto é estimado a partir da diferença entre essas duas variações (Schor; Afonso, 2007).

Para a avaliação de impacto do Pro-Hosp, o grupo de tratamento foi definido como sendo os hospitais da rede SUS de Minas Gerais (públicos e filantrópicos), que participaram do programa entre 2003 e 2008, tendo aderido ao Pro-Hosp em diferentes momentos desse período, ou seja, tendo celebrado contrato e recebido recursos.

Os hospitais do grupo de controle devem apresentar características bastante similares aos hospitais do grupo de tratamento, com a diferença de não terem recebido recurso do Pro-Hosp. Portanto, variações observadas nos indicadores selecionados para a avaliação seriam explicadas apenas pelo recebimento do tratamento, obtendo-se assim o impacto do programa. Devido à forma como o programa foi concebido, não foi possível encontrar em Minas Gerais um grupo de não tratados que seja o mais similar possível aos hospitais beneficiados. Os hospitais públicos e filantrópicos de Minas Gerais que não foram contemplados pelo programa são de porte muito pequeno e apresentam características bastante peculiares. A alternativa escolhida foi definir o grupo de controle a partir do universo de hospitais da rede SUS de estados limítrofes a Minas Gerais: Bahia, Goiás, Rio de Janeiro, São Paulo e Espírito Santo. Para controlar variações específicas ao Estado de Minas Gerais não relacionadas à participação no programa ("efeito estado"), optou-se por incluir também no grupo de controle todos os hospitais mineiros da rede SUS que não foram beneficiados pelo Pro-Hosp.

\section{Seleção dos hospitais para compor o grupo de controle}

Utilizou-se o método de escore de propensão para selecionar os hospitais da rede SUS de estados limítrofes que fossem mais parecidos com os hospitais da rede SUS de Minas Gerais (independentemente de participarem ou não do Pro-Hosp). A utilização dessa metodologia é importante, uma vez que os hospitais dos estados limítrofes podem apresentar características bem distintas às dos de Minas Gerais.

O modelo de escore de propensão foi desenvolvido em Rosenbaum e Rubin (1983), para avaliar o impacto de políticas públicas na ausência de grupos de controle naturalmente randômicos, sendo adaptado para os propósitos da análise realizada no presente estudo. Esse método permite avaliar o impacto desses programas por meio da "aleatorização" da exposição ao tratamento condicional a um vetor de características observáveis $X$. $O$ escore de propensão é a probabilidade individual $\mathrm{P}$ de se receber o tratamento $\mathrm{T}$ condicional a um vetor de características 
observáveis $X$ definidas antes do tratamento. Se a exposição ao tratamento é randômica dentro dos grupos definidos por $X$, segue que a exposição será também randômica dentro dos grupos definidos por $P(X)$. Assim, duas unidades, uma tratada e outra não tratada, terão a mesma distribuição de covariadas se elas têm níveis comparáveis de escore de propensão, sendo possível realizar o pareamento com base apenas nessa probabilidade condicional.

Para este estudo, o escore de propensão é definido como a probabilidade individual $(\mathrm{P})$ de pertencer a Minas Gerais, condicional a um vetor de características observáveis. De acordo com esse método, dois hospitais, um de Minas Gerais e outro de um dos demais estados, terão a mesma distribuição de covariadas, se tiverem níveis comparáveis de escore de propensão. A vantagem desse método é que ele permite reduzir o problema de dimensionalidade no pareamento, quando se tem muitas variáveis a serem analisadas.

O escore de propensão é estimado por meio de um modelo de escolha binária logit, no qual a variável dependente é igual a 1 se o hospital pertence a Minas Gerais (independentemente de ser ou não beneficiado pelo Pro-Hosp), e zero se pertence aos estados limítrofes. O vetor de características observáveis é composto por diferentes indicadores selecionados para análise e apresentados no Quadro 1. Todas as variáveis são medidas para o ano de 2002, exceto o Índice de Desenvolvimento Humano (IDH), cuja última informação disponível refere-se ao ano de 2000.

Quadro 1: Variáveis independentes incluídas no modelo de escore de propensão

\begin{tabular}{|c|c|}
\hline Características & Variáveis independentes \\
\hline \multirow[t]{7}{*}{ Hospitais } & Mortalidade hospitalar \\
\hline & Média dos dias de permanência hospitalar \\
\hline & Taxa de Ocupação Hospitalar \\
\hline & $\begin{array}{l}\text { Proporção de internação por: clínica médica, obstetrícia, pediatria, } \\
\text { cirurgia, causas sensíveis à atenção ambulatorial, Unidade de Trata- } \\
\text { mento Intensivo, alta complexidade e média complexidade }\end{array}$ \\
\hline & Leitos existentes no hospital \\
\hline & Proporção de idosos internados \\
\hline & Idade média dos pacientes internados \\
\hline \multirow[t]{4}{*}{ Municípios } & Índice de Desenvolvimento Humano 2000 (IDH) \\
\hline & Gastos com saúde por habitante \\
\hline & Gastos próprios com saúde por habitante/ Gastos com saúde por habitante \\
\hline & População \\
\hline
\end{tabular}

Fonte: Elaboração própria. 
Após as estimativas, foram selecionados 305 hospitais dos Estados da Bahia, Espírito Santo, Rio de Janeiro, São Paulo e Goiás para compor o grupo de controle. Como o método estimado permite reposição, é possível que um hospital dos estados limítrofes seja controle de mais de um hospital de Minas Gerais (independentemente de terem ou não recebido recursos do Pro-Hosp). Portanto, a amostra de hospitais do grupo de controle foi expandida, considerando o número de vezes que cada hospital foi pareado com os hospitais de Minas Gerais.

\section{Fonte dos dados}

Os dados utilizados neste trabalho são provenientes das seguintes fontes: a) bancos de dados de internações hospitalares do Sistema de Informações Hospitalares do Sistema Único de Saúde (SIH-SUS); b) Cadastro de Hospitais do SIHSUS; c) Cadastro Nacional dos Estabelecimentos de Saúde (CNES); e d) Secretaria de Estado de Saúde de Minas Gerais.

O banco de dados de internações hospitalares do SIH-SUS processa informações para efetuar o pagamento aos hospitais dos serviços prestados pelo SUS, por meio da captação de dados das autorizações de internação hospitalar (AlH). As AlH contêm as informações sobre as características das internações hospitalares realizadas nos hospitais da rede SUS, tais como a principal causa de internação, o procedimento realizado, além de algumas informações sobre o paciente, como idade, sexo e município. O Cadastro de Hospitais do SIH-SUS forneceu informações sobre a quantidade de leitos existentes e de leitos contratados pelo SUS para os anos de 2002 a 2005.

Os dados relativos aos leitos, a partir de 2006, foram extraídos do Cadastro Nacional dos Estabelecimentos de Saúde (CNES). O CNES contém dados relativos às características dos estabelecimentos de saúde existentes no País, vinculados ou não ao SUS, nos aspectos de área física, recursos humanos, equipamentos e serviços ambulatoriais e hospitalares.

A lista dos hospitais participantes do Pro-Hosp foi fornecida pela coordenação do Pro-Hosp e contempla 140 hospitais que receberam recursos em algum ano do programa, entre 2003 e 2008. Dois estabelecimentos foram retirados dessa lista, sendo um que recebeu recurso do Pro-Hosp em 2003/2004 e fechou, e outro que não possuía informações no SIH-SUS para todos os anos. Como consequência, trabalhou-se com 138 hospitais.

\section{Estimação do impacto: modelo de diferenças-em-diferenças}

Considere "Y" um indicador de desempenho, selecionado para a avaliação de impacto do Pro-Hosp. Essa medida pode ser, por exemplo, o Tempo médio de 
permanência hospitalar ou a Taxa de ocupação hospitalar definida para cada um dos hospitais h no grupo de tratamento e de controle em cada ano t. O período de análise varia entre 2002 e 2008, com as entradas dos hospitais no Pro-Hosp ocorrendo nos anos que variam entre 2003 e 2007. Pode-se, então, especificar o seguinte modelo, como definido em Jacobson, Lalonde e Sullivan (1993):

$$
\mathrm{Y}_{\mathrm{ht}}=\alpha_{h}+\gamma_{t} \times U F_{s}+\delta X_{h t}+\partial M_{m t}+\sum_{-3}^{5} \beta_{k} D_{h t}^{k}+\varepsilon_{h t}
$$

Onde:

$$
\begin{aligned}
\alpha_{h}= & \text { efeito fixo, específico de cada hospital } h ; \\
= & \text { conjunto de variáveis binárias para cada ano da análise; } \\
& =\text { conjunto de variáveis binárias representativas de cada unidade da Federação; } \\
X_{h t} & =\text { vetor de variáveis de controle representando características específicas do hospital }
\end{aligned}
$$
$h$ no tempo $t$;

$M_{m t}=$ vetor de variáveis de controle representando características do município $(m)$ no qual o hospital está localizado no tempo t;

$$
\begin{aligned}
& \begin{aligned}
\sum_{-3}^{5} D_{h t}^{k}= & \text { vetor de dummies indicativas da participação do hospital no Pro-Hosp, que } \\
& \text { varia de } 3 \text { anos antes da entrada do hospital no Pro-Hosp, até } 5 \text { anos depois } \\
& \text { que a entrada ocorreu. }
\end{aligned} \\
& \begin{aligned}
\mathcal{E}_{h t}=\text { termo de erro; } \\
=\text { coeficientes estimados. }
\end{aligned}
\end{aligned}
$$

$\beta_{k}$ é vetor de coeficientes estimados que indica o efeito médio do tratamento sobre o tratado no ano t, ou seja, fornece o impacto do Pro-Hosp sobre o desempenho dos hospitais antes e após a adesão. Com essa especificação, é possível avaliar: (a) melhorias no desempenho dos hospitais após a implementação do programa; (b) por quanto tempo essas melhorias se mantiveram após a implementação; e (c) se houve alguma alteração nesses indicadores antes mesmo de o programa ser implementado.

Os modelos foram estimados utilizando dados em painel. Os efeitos fixos para cada hospital controlam as características não observáveis dos hospitais, que são constantes ao longo do tempo e que poderiam estar correlacionadas com a adesão do hospital ao Pro-Hosp, como qualidade da gestão, por exemplo. Para verificar a presença de correlação serial de primeira ordem dos resíduos, realizou-se o teste de Wooldridge para dados em painel (Wooldridge, 2002). No caso de o resultado do teste rejeitar a hipótese Ho de que não há correlação serial de primeira ordem, o modelo com dados em painel de efeito fixo foi estimado corrigindo-se para a presença de correlação serial.

\section{Variáveis dependentes e independentes}

Para avaliar o impacto, foram selecionados quatro indicadores de desempenho do programa (variáveis dependentes): o tempo médio de permanência nos 
hospitais (TMP), a taxa média de ocupação hospitalar (TO), a taxa média de mortalidade hospitalar (TMH) e a proporção de cesáreas (PC).

O tempo médio de permanência (TMP) é definido pelo quociente entre o número de dias de internação em um dado período t e o número de internações no mesmo período:

$T M P=\frac{D I_{t}}{N I_{t}}$

Onde:

$\mathrm{DI}=$ total de dias de internação, sendo computados os dias entre a internação e a alta.

$\mathrm{NI}=$ número de internações.

$t=$ ano com $t=2002,2003, \ldots ., 2008$.

A taxa média de ocupação hospitalar (TO) é definida como sendo igual ao quociente entre o número de dias de internação em um dado período t e o número de leitos hospitalares disponíveis no mesmo período t. Neste artigo, o cálculo dessa taxa levou em consideração o número de meses que o hospital integrou a rede SUS. Algebricamente:

$$
T O_{t}=\frac{D I_{t}}{(N M * 30) * L H_{t}}
$$

Onde:

DI = dias de internação no ano.

$\mathrm{NM}=$ número de meses que o hospital integrou a rede SUS no ano.

$\mathrm{LH}=$ número de leitos hospitalares.

$\mathrm{t}=$ ano com $\mathrm{t}=2002,2003, \ldots ., 2008$.

A taxa de mortalidade hospitalar (TMH) constitui um indicador tradicional do desempenho do hospital, e é definida como a razão entre o número de óbitos num dado período $t$ em relação ao número de internações no mesmo período, multiplicada por 100. Algebricamente, a TMH pode ser representada como:

$$
\begin{aligned}
& \mathrm{TMH}_{t}=\frac{O H_{t}}{A H_{t}} \times 100 \\
& \text { Onde: } \\
& \mathrm{OH}=\text { óbitos hospitalares. } \\
& \mathrm{AH}=\text { total de internações hospitalares. } \\
& \mathrm{t}=\text { ano com } \mathrm{t}=2002,2003, \ldots . ., 2008 .
\end{aligned}
$$

A proporção de cesáreas (PC) é definida pelo quociente entre o total de partos cirúrgicos realizados em um dado período t e o total de partos ocorridos no mesmo período. 
Algebricamente, tem-se que:

$P C=\frac{P C I_{t}}{N P_{t}}$

Onde:

$\mathrm{PCl}=$ número de partos cirúrgicos.

$\mathrm{NP}=$ número de partos.

$\mathrm{t}=$ ano com $\mathrm{t}=2002,2003, \ldots ., 2008$.

As variáveis independentes incluídas no modelo referem-se às características hospitalares. Consideraram-se também informações sobre os gastos com saúde municipais (totais e próprios) disponíveis no Sistema de Informação em Orçamentos Públicos de Saúde (SIOPS). Todas essas medidas foram incluídas com defasagem temporal de um ano (Quadro 2).

Quadro 2: Variáveis independentes incluídas no modelo de análise

\begin{tabular}{|c|c|}
\hline Características & Variáveis independentes \\
\hline \multirow[t]{2}{*}{ Municípios } & Gastos com saúde por habitante. \\
\hline & $\begin{array}{l}\text { Gastos próprios com saúde por habitante/Gastos com saúde por } \\
\text { habitante. }\end{array}$ \\
\hline \multirow[t]{6}{*}{ Hospitais } & Proporção dos leitos existentes que são contratados pelo SUS. \\
\hline & $\begin{array}{l}\text { Proporção de internação por: clínica médica, obstetrícia, pediatria, } \\
\text { cirurgia, causas sensíveis à atenção ambulatorial, UTI, alta comple- } \\
\text { xidade e média complexidade. }\end{array}$ \\
\hline & Leitos existentes no hospital. \\
\hline & Meses de participação no SUS. \\
\hline & Proporção de idosos internados. \\
\hline & Idade média dos pacientes internados. \\
\hline
\end{tabular}

Fonte: Elaboração própria.

Não foi possível incluir na análise características socioeconômicas e demográficas dos municípios de localização do hospital, devido à indisponibilidade dessas informações medidas em nível municipal ao longo do tempo. 


\section{Resultados}

\section{Análise descritiva dos indicadores de desempenho dos hospitais participantes do Pro-Hosp}

O comportamento dos indicadores de desempenho hospitalar selecionados para a avaliação de impacto do Pro-Hosp foi analisado para o período de 2002 a 2008 e os resultados são apresentados na Tabela 1.

Analisando a taxa média de ocupação total dos hospitais participantes do ProHosp, uma primeira questão a ser ressaltada é a significativa redução (18\%) ocorrida entre 2002 e 2003, quando passou de 62,15\% para 50,97\%. Quando se considera o período 2003 a 2008, no entanto, constata-se uma evolução positiva da taxa média de ocupação total, que passa de 51\% para 59\%. Tem-se, portanto, que, na vigência do Pro-Hosp, a taxa média de ocupação total dos hospitais participantes elevou-se quase $16 \%$.

No Brasil, a taxa média de ocupação dos hospitais da rede SUS foi de $48 \%$ em 1999, tendo variado de $24 \%$ no Maranhão a 74\% no Distrito Federal (BRASIL, 2002). Do cálculo desse indicador realizado por Marinho, Moreno e Cavalini (2001), também para o ano de 1999, resultou para Minas Gerais o percentual de 44\%, um pouco inferior à média nacional. Ainda de acordo com esses autores, a média das taxas de ocupação foi de $64 \%$ nas regiões metropolitanas e de $62 \%$ nas capitais dos estados. A média da Região Metropolitana de Belo Horizonte foi de $61 \%$ e dos hospitais localizados em Belo Horizonte, de $67 \%$.

Tabela 1: Indicadores de desempenho dos hospitais participantes do Pro-Hosp 2002 a 2008

\begin{tabular}{|c|c|c|c|c|c|c|c|c|c|}
\hline \multirow{2}{*}{$\begin{array}{l}\text { Indicadores de } \\
\text { desempenho } \\
\text { hospitalar }\end{array}$} & \multicolumn{7}{|c|}{ Período } & \multicolumn{2}{|c|}{ Variação } \\
\hline & 2002 & 2003 & 2004 & 2005 & 2006 & 2007 & 2008 & $\begin{array}{c}2002 / \\
2008\end{array}$ & $\begin{array}{r}2003 / \\
2008\end{array}$ \\
\hline Taxa de ocupação total & 62,15 & 50,97 & 53,74 & 52,10 & 53,51 & 54,14 & 58,92 & $-5,2$ & 15,6 \\
\hline Mortalidade geral & 3,29 & 3,50 & 3,66 & 3,71 & 3,86 & 4,06 & 4,04 & 22,7 & 15,3 \\
\hline $\begin{array}{l}\text { Tempo médio de } \\
\text { permanência total }\end{array}$ & 4,20 & 4,19 & 4,26 & 4,16 & 4,16 & 4,21 & 4,37 & 3,8 & 4,2 \\
\hline Proporção de cesáreas & 30,06 & 30,84 & 32,65 & 33,84 & 34,87 & 36,24 & 38,38 & 27,7 & 24,4 \\
\hline
\end{tabular}

Fonte: Sistema de Informações Hospitalares do Sistema Único de Saúde (SIH-SUS); Cadastro de Hospitais do SIH-SUS, Cadastro Nacional dos Estabelecimentos de Saúde (CNES).

Parece haver consenso de que uma taxa média de ocupação ótima seja por volta dos $85 \%$. Taxas abaixo de $80 \%$ são consideradas ineficientes e as superiores a $90 \%$ colocam em risco a capacidade do hospital de atender um aumento inesperado da 
demanda (BAgust, Place e Posnetr, 1999). Na medida em que as doenças e os acidentes não são previsíveis, é necessário que os hospitais mantenham certa capacidade de reserva para atendimentos de emergência e urgência (HEMESATH e POPE, 1989). Elevadas taxas de ocupação associam-se ainda a piores resultados no controle de infecções e a maiores taxas de mortalidade. Desse modo, considerando as taxas de ocupação encontradas nos hospitais Pro-Hosp, pode-se dizer que essas ainda têm espaço para serem ampliadas.

A análise mostrou, com base em informações não apresentadas neste artigo, mas realizadas durante o estudo, que embora a taxa média de ocupação total dos hospitais do Pro-Hosp tenha-se mostrado abaixo do desejável em 2008 (59\%, enquanto a portaria 1101/GM/2002 do Ministério da Saúde estabelece que a média desejável seja de $80 \%$ a $85 \%$ ), o mesmo não se pode dizer das taxas das clínicas médica e pediátrica que foram de $81 \%$ e $92 \%$ respectivamente, no mesmo ano. Para efeitos de comparação, a taxa média de ocupação dos hospitais da rede SUS no País foi de $48 \%$ em 1999, tendo variado de $74 \%$ no Distrito Federal a $24 \%$ no Maranhão. Já nos países da Organização para Cooperação e Desenvolvimento Econômico (OCDE), essa taxa média foi de $72 \%$ em 2002, sendo considerável a variação entre eles (LA FoRGIA; Couttolenc, 2009).

Entre os hospitais participantes do Pro-Hosp, a taxa média de mortalidade geral passou de 3,29\% em 2002 para 4,04\% em 2008, o que representou uma variação positiva de $22,7 \%$. Analisando essa medida por grupo de idade e sexo, observouse que, tanto para homens quanto para mulheres, a taxa de mortalidade hospitalar, como esperado, apresenta um formato de $U$, sendo mais elevada nos grupos de idade extremos.

No Brasil, a mortalidade hospitalar apresentou uma média de 2,63\% do total das altas hospitalares em 1999 , tendo variado de 4,05\% no Rio de Janeiro a 0,88\% no Maranhão (BRASIL, 2002). A respeito dessa diferença, é possível que ela reflita a maior complexidade dos casos tratados. Não se pode descartar, também, diferenças na qualidade das informações.

De acordo com Travassos, Noronha e Martins (1999), diversos estudos têm apontado que a mortalidade hospitalar varia acentuadamente entre hospitais. No entanto, quando buscam explicar as causas dessa variação, produzem resultados contraditórios. Tais variações podem ser atribuídas a diferenças quanto à gravidade do estado de saúde dos pacientes, variações na eficácia das tecnologias médicas empregadas, adequação do processo de cuidado ao paciente, e a erros aleatórios. Por fim, em que pese a existência de questões conceituais e metodológicas que 
devem ser aprimoradas, Travassos, Noronha e Martins (1999) argumentam que o uso das taxas de mortalidade hospitalar permite uma primeira aproximação aos problemas com a qualidade dos cuidados prestados e, por isso, pode ser considerada uma forma capaz de discriminar hospitais com desempenho diferenciado.

No que diz respeito ao tempo médio de permanência nos hospitais participantes do Pro-Hosp, observou-se uma evolução de 4,2 dias em 2002 para 4,4 dias em 2008, o que representou uma elevação discreta de aproximadamente $4 \%$. Ainda que tenha havido uma variação positiva no tempo médio de permanência nos hospitais participantes do Pro-Hosp, deve-se destacar que esse indicador permaneceu, ao longo do período, abaixo da média do País e do conjunto dos hospitais da rede SUS de Minas Gerais: 5,98 e 5,82 dias respectivamente (BRASIL, 2002; Marinho, Moreno e Cavalini, 2001). O tempo médio de permanência hospitalar, para o Brasil e para Minas Gerais, em especial para os hospitais Pro-Hosp, está abaixo daquele observado para os países mais desenvolvidos. Ressalta-se, contudo, que, como essas medidas não estão padronizadas por idade e sexo, essa diferença pode estar refletindo diferenças na estrutura etária da população.

Considerando-se a proporção média de cesáreas nos hospitais participantes do Pro-Hosp, ela foi de 30,1\% em 2002, e, após elevar-se gradualmente, atingiu $38,4 \%$ em 2008, o que representou uma variação positiva de $27,6 \%$.

No Brasil, a proporção de cesáreas sofre variações entre os sistemas público e privado. Enquanto no SUS, responsável por quase $88 \%$ dos partos, essa proporção foi de $27,53 \%$ em 2004 , no sistema de saúde suplementar ela foi de $79,7 \%$, no mesmo ano (ANS, 2008). Em Minas Gerais, a Deliberação da Comissão Intergestores Bipartite (CIB/MG), de abril de 2001, aprovou os limites percentuais de partos cesáreos para as unidades hospitalares do SUS/MG, para o período de 2000 a 2008. A análise da evolução das proporções pactuadas com 536 hospitais indica uma intenção de reduzi-las de uma média de aproximadamente 33\% em 2000, para cerca de $22 \%$ em 2008.

\section{Resultados da avaliação de impacto}

Esta seção apresenta os resultados da avaliação de impacto do Pro-Hosp nos indicadores de desempenho selecionados, conforme Tabela 2.

De acordo com a Tabela 2, os resultados mostram um efeito significativo do Pro-Hosp sobre a proporção média de cesáreas. Esse efeito é importante um ano após a entrada do hospital no programa, o que permaneceu nos anos subsequentes. 
Conforme esperado, o Pro-Hosp contribuiu para reduzir a proporção média de cesáreas dos hospitais participantes e esse efeito foi sentido até cinco anos depois. Tendo em vista que a análise descritiva indicou uma elevação da proporção de cesáreas de 27,6\% entre 2002 e 2008, nos hospitais participantes do Pro-Hosp, podese concluir que, na ausência do programa, a variação positiva nesse indicador teria sido ainda maior.

Tabela 2: Efeito do Pro-Hosp na taxa de ocupação hospitalar, na mortalidade hospitalar, no tempo médio de permanência e na proporção de cesáreas

\begin{tabular}{l|c|c|c|c}
\multicolumn{1}{c|}{ Efeito do Pro-Hosp } & $\begin{array}{c}\text { Taxa de } \\
\text { ocupação } \\
\text { hospitalar }\end{array}$ & $\begin{array}{c}\text { Mortalidade } \\
\text { hospitalar }\end{array}$ & $\begin{array}{c}\text { Tempo médio } \\
\text { de } \\
\text { permanência }\end{array}$ & $\begin{array}{c}\text { Proporção de } \\
\text { cesáreas }\end{array}$ \\
\hline 3 anos antes & $-0,06$ & $-0,01$ & $-0,06$ & $-0,03$ \\
2 anos antes & $-0,02$ & $-0,01$ & 0,21 & $-0,08$ \\
1 ano antes & 0,03 & $-0,01$ & 0,29 & $-0,13$ \\
ano que recebeu recurso & & & & $-0,16$ \\
pela 1a vez & 0,06 & 0,00 & 0,25 & $-0,18^{*}$ \\
1 ano depois & 0,09 & 0,00 & 0,32 & $-0,18$ \\
2 anos depois & 0,10 & 0,00 & 0,31 & $-0,20^{*}$ \\
3 anos depois & 0,12 & 0,00 & 0,34 & $-0,20^{*}$ \\
4 anos depois & 0,14 & 0,00 & 0,34 & $-0,19^{*}$ \\
5 anos depois & 0,16 & 0,00 & 0,33 & 3856 \\
Número de observações & 4868 & 4869 & 5844 &
\end{tabular}

Fonte: Sistema de Informações Hospitalares do Sistema Único de Saúde (SIH-SUS), Cadastro de Hospitais do SIH-SUS e Cadastro Nacional dos Estabelecimentos de Saúde (CNES).

Nota: ${ }^{*}=$ significativo.

Para a taxa de ocupação total, mortalidade hospitalar e tempo médio de permanência nos hospitais, o impacto do programa não se mostrou significativo. Ou seja, não houve diferença estatisticamente significativa entre a variação nesses três indicadores, antes e depois do Pro-Hosp, para os hospitais que participaram do programa, e a variação observada para os hospitais que não participaram do Pro-Hosp, no mesmo período.

A avaliação externa do primeiro ano do Pro-Hosp macrorregional, realizada pela Fundação João Pinheiro em 2005, constatou que alguns diretores de hospitais viram na iniciativa uma oportunidade de retomada de investimentos necessários e constantemente adiados dada à crise financeira; outros viram no programa a possibilidade de recursos para custeio de despesas correntes (Fundação João PINHEIRo, 2005). Nessas circunstâncias, o aumento da taxa de ocupação pode ter sido postergado para um segundo momento, o que teria sido favorecido, ainda, pelas condições nas quais as pactuações dos compromissos e metas foram feitas. Como não houve diagnóstico prévio da situação de cada hospital antes do início do programa, que pudesse fornecer parâmetros razoáveis, cada hospital registrou 
no Plano de Ajustes e Metas a situação inicial e a meta a ser alcançada, sem uma avaliação dos valores correspondentes aos indicadores de desempenho que se propunham melhorar, por parte da Secretaria de Estado da Saúde. Por fim, e dado que o aumento da taxa de ocupação não depende apenas do gestor do hospital, mas também do número de autorizações de internação hospitalar (AlH) de que dispõe o hospital, não se sabe até que ponto os hospitais podem ter enfrentado problemas com relação a essa questão.

No que diz respeito à mortalidade hospitalar e ao tempo médio de permanência nos hospitais, a ausência de um impacto significativo do Pro-Hosp pode refletir as diferenças na gravidade dos casos, uma vez que o modelo não controla as condições de saúde dos pacientes internados em cada hospital. A análise descritiva mostrou que houve um aumento da taxa média de mortalidade geral nos hospitais participantes do programa, sendo esse aumento mais acentuado entre os indivíduos acima de 45 anos. Dadas as dificuldades de trabalhar com as informações referentes à mortalidade, acredita-se que estudos adicionais precisam ser realizados para tentar entender melhor e justificar os resultados encontrados.

\section{Considerações finais}

Este estudo apresentou os resultados da avaliação do Programa de Fortalecimento e Melhoria da Qualidade dos Hospitais do SUS-MG (Pro-Hosp). Para avaliar o impacto do programa, foram selecionados quatro indicadores de desempenho hospitalar: a proporção média de cesárea, a taxa média de ocupação, a taxa média de mortalidade e o tempo médio de permanência. Como enfatizado, os indicadores selecionados constituem alguns dos inúmeros compromissos e metas que os hospitais se comprometeram a cumprir ao aderir ao programa. Portanto, os resultados devem ser compreendidos nesse contexto.

Constatou-se que o Pro-Hosp teve impacto na proporção média de cesáreas no sentido esperado, ou seja, de reduzi-las. A despeito de essa proporção ter aumentado de 30\% em 2002 para 38\% em 2008, os resultados sugerem que, na ausência do programa, o aumento teria sido maior. Vale lembrar que as taxas de cesáreas no Brasil estão entre as mais altas do mundo, e vêm aumentando tanto no SUS quanto no setor de saúde suplementar. Para enfrentar essa situação, os hospitais da rede SUS têm sido estimulados a diminuir esse percentual. No caso específico dos de Minas Gerais, eles se propuseram a reduzir de uma média de aproximadamente 33\% em 2000 para cerca de 22\% em 2008. Constata-se, portanto, que, pelo menos em termos médios, essa meta ainda está longe de ser alcançada. 
Para os demais indicadores de desempenho, os resultados não se mostraram significativos. Ou seja, não houve diferença estatisticamente significativa entre as variações observadas nesses indicadores, antes e depois do Pro-Hosp, para os hospitais que aderiram ao programa, e a variação observada para os hospitais com características semelhantes, mas localizados na Bahia, Espírito Santo, Goiás, Rio de Janeiro e São Paulo, no mesmo período. No entanto, é importante fazer algumas considerações de modo a qualificar esses resultados.

No que se refere ao tempo médio de permanência total, observou-se que ele se manteve relativamente constante entre 2002 e 2008, quando foi de 4,4 dias. Isso significa que esse indicador se manteve abaixo da média do País e do conjunto dos hospitais da rede SUS de Minas Gerais, que era de 5,98 e 5,8 dias respectivamente, em 1999. O tempo médio de permanência hospitalar nos hospitais do Pro-Hosp está abaixo também daquele observado para a média dos países da OCDE (7,8 dias em 2002), ainda que essa diferença possa estar refletindo diferenças na estrutura etária da população (LA ForgIA; CoUtTolenc, 2009). Outras avaliações, não apresentadas neste artigo, mas realizadas durante o estudo, mostraram que o tempo de permanência nas clínicas cirúrgica, médica, pediátrica e obstetrícia dos hospitais Pro-Hosp também se mostrou abaixo dos parâmetros estabelecidos pelo Ministério da Saúde em 2001. Porém, da mesma forma, quando avaliado separadamente por setor de internação, não foi observado o impacto.

Essas informações sugerem que o tempo de permanência nos hospitais que aderiram ao Pro-Hosp já esteja, pelo menos em termos médios, num patamar difícil de ser reduzido sem colocar em risco a qualidade da assistência. $O$ fato de não ter sido feito um diagnóstico prévio da situação de cada hospital antes do início do programa, que pudesse fornecer parâmetros razoáveis para orientar as pactuações dos compromissos, talvez possa explicar por que a redução desse indicador esteja entre as metas do programa.

No caso da taxa de ocupação, foi ressaltado que o aumento da taxa de ocupação não depende apenas do gestor do hospital, mas também do número de autorizações de internação hospitalar (AIH) de que dispõe o hospital, e que alterar esse número não é uma questão trivial.

Outro ponto que deve ser considerado, e que vale também para outros indicadores de desempenho, é que o cumprimento das metas estabelecidas pode depender de outras ações, como investimentos que, exatamente por isso, podem ter sido priorizados. Nesse contexto, o enfrentamento de questões mais complexas, como a taxa de ocupação, pode ter sido postergado, ainda mais quando se consideram as reconhecidas dificuldades enfrentadas pelos hospitais da rede SUS, como o endividamento e a baixa capacidade de investimento. 
A respeito da mortalidade hospitalar, foi destacado que, como não foi possível controlar pelo grau de gravidade dos casos, a ausência de impacto pode estar refletindo, entre outros aspectos, a maior complexidade dos casos tratados. Em uma análise específica por setor de atendimento, constatou-se que, assim como analisado em conjunto, as taxas de mortalidade das clínicas cirúrgica e médica aumentaram de 2002 a 2008. A elevação mais expressiva da taxa de mortalidade na clínica médica e o aumento, também relativamente maior, do tempo de ocupação nessa clínica poderiam significar que o Pro-Hosp estaria favorecendo o acesso de pacientes que, de outro modo, morreriam em casa, sem assistência. Isso posto, pode-se concluir que os resultados da avaliação de impacto do Pro-Hosp nos indicadores de desempenho considerados ainda precisam ser melhor compreendidos, daí a importância da análise de processo. Em que pese tal fato, acredita-se que eles jogam luz sobre o desempenho dos hospitais participantes do Pro-Hosp nos aspectos considerados, e constituem um primeiro passo para se criar um intercâmbio entre execução, avaliação e reorientação.

Finalmente, a despeito das dificuldades de avaliar programas públicos, principalmente devido à dificuldade de obter dados, acredita-se que esta seja uma contribuição inicial tanto para avaliação do programa, como um exercício de avaliação de impacto de políticas públicas, empregando o método diferençasem-diferenças e o pareamento para obtenção do grupo de controle. Sabe-se que as avaliações, independentemente de sua natureza, devem estar presentes nas diversas fases de implantação de programas e políticas públicas. Afinal, o processo de implementação não consiste apenas da simples operacionalização das atividades previamente estabelecidas. Ao contrário, trata-se de um processo bem mais complexo que, além de ter que responder a dificuldades não previstas, constitui-se em processo marcado por mudanças e adaptações, que se impõem à tomada de novas e importantes decisões. Particularmente, programas inovadores, como é o caso do Pro-Hosp, dependem, entre outros fatores, de mudanças nas hierarquias de prioridades, nas práticas e culturas organizacionais, no comportamento tanto do público-alvo quanto dos implementadores.

\section{Referências bibliográficas}

Agência Nacional de Saúde Suplementar (ANS). O modelo de atenção obstétrica no setor de Saúde Suplementar no Brasil : cenários e perspectivas. Rio de Janeiro: ANS, 2008. 158 p.

Bagust, A., Place, M., Posnett, J. W. Dynamics of bed use in accommodating emergency admissions: stochastic simulation model. BMJ, v. 319, p.155-158, July, 1999.

BItTAR, O. J. N. V. Indicadores de qualidade e quantidade em saúde. Revista de administração em saúde, v. 3, n.12, p. 21-28, 2001. 
Brasil. Ministério da Saúde. Portaria n. 1101/GM de 2002. Parâmetros Assistenciais. Brasília, 2009.

DonabediAn, A. The Definition of Quality and Approaches to its Assessment. Health Administration Press, Ann Arbor, 1980. 163p.

Fundação João Pinheiro. Estudo de avaliação externa do Programa de Fortalecimento e Melhoria da Qualidade dos Hospitais do SUS/MG: módulo macrorregional. Belo Horizonte, 2005.

Hemesath, M., Pope, G. C. Linking Medicare capital payments to hospital occupancy rates. Health Affairs, p.104-116, Fall, 1989.

Jacobson, L., Lalonde, R., Sullivan, D. Earnings Losses of Displaced Workers. American Economic Review, v. 83, n. 4, p. 685-709, 1993.

La Forgia, G. M., Couttolenc, B. F. Desempenho hospitalar no Brasil em busca de excelência. São Paulo: Singular, 2009.

Marinho, A., Moreno, A. B., CAvalini, L. T. Avaliação descritiva da rede hospitalar do sistema único de saúde (SUS). Rio de Janeiro: IPEA, 2001. (Texto para Discussão, 848).

Minas Gerals. Secretaria de Estado de Saúde. Manual do Pro-Hosp. Belo Horizonte, 2007A.

Secretaria de Planejamento e Gestão. Plano Mineiro de Desenvolvimento Integrado. Belo Horizonte, 2003.

Secretaria de Planejamento e Gestão. Plano Mineiro de Desenvolvimento Integrado 2007-2023. Belo Horizonte, 2007B.

Secretaria de Planejamento e Gestão. Plano Mineiro de Desenvolvimento Integrado 2007-2023. Belo Horizonte, 2011.

NeRI, M. C.; SoARES, W. L. Estimando o impacto da renda na saúde através de programas de transferência de renda aos idosos de baixa renda no Brasil. Cad. Saúde Pública, v. 23, n. 8, p. 1845-1856, 2007.

Peixoto, B.; Allanic, L. R.; Souza, L. M.; Lima, L. L.; Camargos, M. C. S.; Menezes-Filho, N. A. Avaliação do programa de redução da mortalidade infantil e materna Viva Vida (PVV). Relatório Final. Belo Horizonte: FJP, 2010.

Pereira, G. C. Uma Avaliação de Impacto do Programa Mais Educação no Ensino Fundamental. Dissertação de Mestrado. Instituto de Economia. UFRJ. 2011.

Rosenbaum, P. R., RuBin, D. B. The central role of the propensity score in observational studies for causal effects. Biometrika, v.70, n.1, p. 41-55, 1983.

Schor, A. Afonso, L. E. Avaliação Econômica de Projetos Sociais. 2ạ. ed. São Paulo: Fundação Itaú Social, 2007. 52p.

Souza L. M., Pereira, D. R. M., Delgado, V. M. S. Avaliação de impacto da ação de lavoura do programa Minas Sem Fome sobre a renda bruta domiciliar per capita dos agricultores familiares de Minas Gerais. In: Encontro Anual da Associação Nacional dos Programas de Pós-Graduação em Administração, 35으 Anais... Rio de Janeiro: ANPAD, 2011.

Travassos, C., Noronha, J. C., Martins, M. Mortalidade hospitalar como indicador de qualidade: uma revisão. Ciência \& Saúde Coletiva, v. 4, n. 2, p. 367-381, 1999. 
Vaitsman, J.; Paes-Sousa, R. (org.) Avaliação de políticas e programas do MDSResultados: Bolsa Família e Assistência Social. Ministério do Desenvolvimento Social e Combate à Fome. Brasília, DF: MDS, v. 2, 2007.

WooldRIDGe, J. M. Econometric Analysis of cross-section and panel data. London: The MIT Press, 2002.

Kenya Valeria Micaela de Souza Noronha

É doutora em Economia pela Universidade Federal de Minas Gerais (UFMG) e professora adjunta II da UFMG. Contato: knoronha@cedeplar.ufmg.br

Fátima Beatriz Carneiro Teixeira Pereira Fortes

É doutora em Ciência Política pela Universidade Federal de Minas Gerais (UFMG) e pesquisadora em Ciência e Tecnologia da Fundação João Pinheiro. Contato: fatima.fortes@fjp.mg.gov.br

Mirela Castro Santos Camargos

É doutora em Demografia pela Universidade Federal de Minas Gerais (UFMG) e pesquisadora em Ciência e Tecnologia da Fundação João Pinheiro. Contato: mirela.camargos@fjp.mg.gov.br

Danielle Ramos de Miranda Pereira

É doutora em Demografia pela Universidade Federal de Minas Gerais (UFMG) e pesquisadora em Ciência e Tecnologia da Fundação João Pinheiro. Contato: danielle.pereira@fjp.mg.gov.br 
